differently with few children presenting with elevated IgG4 levels. Management is also unclear with limited evidence to suggest any management strategy superior to another or the long-term outcomes of paediatric patients.

Aim To present a case of possible autoimmune pancreatitis, presenting with obstructive jaundice, managed without steroids.

Subject: A 12-year-old Caucasian male presented with a week of pruritus and bruising with a 48 -hour history of diarrhoea and jaundice. There was no history of abdominal pain, weight loss or fatigue. He was previously fit and well with no significant family history. Examination revealed petechiae and ecchymosis but no positive abdominal findings.

Results At presentation bloods showed bilirubin 277 umol/L (201 umol/L conjugated), alanine transaminase 88 unit/L, alkaline phosphatase 802 unit/L, gamma glutamyltransferase 79 unit/L and international normalised ratio 0.9. Amylase and lipase were normal throughout. Abdominal ultrasound at the presenting hospital revealed a $2.5 \mathrm{~cm}$ mass at the head of the pancreas with a dilated gallbladder.

The child underwent a MRCP which showed a $3 \mathrm{~cm}$ mass at the pancreatic head with marked intra and extrahepatic biliary dilatation. The pancreatic duct was not visible in the pancreatic head. Endoscopic ultrasonography confirmed a diffusely abnormal pancreas with no pancreatic duct dilatation. There was no discrete mass visible. An ERCP revealed a smooth single stenosis of $20 \mathrm{~mm}$ in the distal and mid common bile duct with pre-stenotic dilatation. A stent was inserted.

Autoantibodies were normal, as were immunoglobulins, including IgG4. Hepatitis serology and alpha-fetoprotein were also normal. A pancreatic biopsy was taken which showed mild chronic inflammation, minor acinar atrophy with intralobular oedema and some fibrosis. Immunohistochemistry for IgG4 disease was negative.

Maximal bilirubin was 334, alkaline phosphatase 869 and alanine transaminase 81 prior to stent insertion. These were falling prior to stent insertion however fell rapidly post insertion and were within normal limits 4 weeks post stent insertion. The patient received no further medical management and is due for repeat imaging prior to proposed stent removal in the next few weeks.

Summary We present a 12-year-old boy who presented with typical radiological findings of presumed autoimmune pancreatitis. He was managed endoscopically with stent insertion and achieved a rapid resolution in symptoms and biochemical markers. Autoimmune screening was negative and there were no positive diagnostic findings with regard to IgG4. The child did not receive steroid therapy and is awaiting repeat imaging prior to proposed stent removal.

Conclusion Autoimmune pancreatitis is a rare condition in children and management is often extrapolated from adult guidelines. Collaborative working is required for the creation of paediatric guidelines to guide further management of this rare condition.

\section{P02 A CHILD WITH AN ANTENATAL DIAGNOSIS OF A CYST IN ABDOMEN - A CASE REPORT}

Muhammad Ghias, Win Zaw. Northampton General Hospital NHS Trust

10.1136/flgastro-2021-bspghan.13

Objectives and Study To describe a case which posed difficulties in diagnosing an infant with biliary atresia.
Methods and Results A term female infant was born by normal delivery with no significant perinatal events. The antenatal scan had showed a cystic abdominal mass $(9 \times 8 \times 10 \mathrm{~mm})$ of unknown origin.

An ultrasound scan was done on Day 4 of life. It showed an $8 \times 9 \times 8 \mathrm{~mm}$ very well-defined spherical fluid filled mass lying in the position of the second part of the duodenum. This was adjacent to but not apparently arising from the head of the pancreas. Radiologically it was thought to be a duplication cyst.

She continued to be fed well. She was seen at the clinic on Day 27 for prolonged jaundice. Her total bilirubin was 152 $\mu \mathrm{mol} / \mathrm{l}$ with conjugated bilirubin of $120.5 \mu \mathrm{mol} / \mathrm{land}$ ALT of 54IU/L. Further investigations were done.

Ultrasound scan on Day 30 showed similar findings to the previous one. The cyst was adjacent to the duct but not connected. There was no dilatation of bile duct.

MRCP was done on Day 34. It showed a $6 \mathrm{~mm}$ cyst adjacent to distal CBD and no connection between the cyst and the duct. Intrahepatic and CBD were not dilated.

Serial LFT were showed high level of conjugated fraction of bilirubin as well as gamma GT. initially the stools were pigmented but they became pale and urine became darker on Day 29.

She was then transferred to the Tertiary Liver unit where she underwent liver biopsy which showed plugs of bile, canalicular cholestasis and periductal fibrosis.

On Day 41, she underwent surgery for Kasai procedure. The intraoperative cholangiogram showed a cystic structure with no connection with the biliary tree. Intraoperative findings were suggestive of biliary atresia.

Abstract P02 Table 1
\begin{tabular}{l|l|l|l|l|l|l|}
$\begin{array}{l}\text { Age } \\
\text { (day }\end{array}$ & $\begin{array}{l}\text { Bilir } \\
\text { ubin }\end{array}$ & Coni & ALT & AST & ALP & GGT \\
s) & & & & & & \\
\hline 27 & 152 & 120 & 54 & - & 365 & - \\
\hline 28 & 157 & 121 & 55 & - & 391 & 1693 \\
\hline 29 & 163 & 129 & 57 & 103 & 390 & 1656 \\
\hline 31 & 156 & 124 & 47 & 91 & 374 & 1303 \\
\hline 33 & 159 & 127 & 48 & 94 & 396 & 1171 \\
\hline
\end{tabular}

Conclusion The presence of a cystic structure at the porta hepatis without intrahepatic biliary ductular dilatation goes towards the diagnosis of biliary atresia, in the antenatal period. Cassacia et al found that anechoic small cyst in the hepatic hilum is highly suspicious of BA.

Presence of a triangular cord sign which is the visualisation of the fibrotic cord in the portal hilum is one of the hallmarks of sonographic imaging with a positive predictive value of $95 \% 4$. We were not able to identify this sign. The utility of MRCP has not been encouraging in view of the cost, varying results and the need for immobilisation.

This case has demonstrated to have a low threshold of diagnosing $\mathrm{BA}$ in the presence of antenatal diagnosis of cyst at the porta hepatis and early referral to the tertiary centre is advisable. 